\title{
A positron trap and beam apparatus for atomic and molecular scattering experiments
}

\author{
J. P. Sullivan, A. Jones, P. Caradonna, C. Makochekanwa, and S. J. Buckman \\ Centre for Antimatter-Matter Studies, Research School of Physical Sciences and Engineering, \\ Australian National University, Canberra, Australia
}

(Received 13 August 2008; accepted 24 October 2008; published online 21 November 2008)

\begin{abstract}
An instrument has been designed and constructed to provide new insights into fundamental, low energy positron scattering processes. The design is based on the Surko trap system and produces a pulsed positron beam with an energy resolution of as good as $54 \mathrm{meV}$. The design and operation of the apparatus is explained, while the first experimental results from this apparatus have been demonstrated in recent publications. () 2008 American Institute of Physics.
\end{abstract}

[DOI: $10.1063 / 1.3030774]$

\section{INTRODUCTION}

Positron atomic physics has long been hampered by the lack of intensity and energy resolution available in conventional positron beams when compared to those available for electron scattering experiments. ${ }^{1}$ However the use of a buffer gas trap in a magnetic field to store and cool positrons and then, subsequently, to produce a pulsed positron beam has revolutionized the field. This has made available, for the first time, a tool that allows the exploration of detailed state selective processes in positron scattering. ${ }^{2-5}$ The apparatus described in this paper has been designed to take advantage of the techniques of trapping and scattering in a strong magnetic field (typically scattering experiments with positrons have used only a weak magnetic field of less than $10 \mathrm{G}$ ) and to apply them to fundamental scattering measurements. While there are other apparatuses built to take advantage of the scheme devised by the UC San Diego group, ${ }^{6,7}$ this is the first that has been designed specifically for the measurement of low energy positron scattering and has several new aspects to the design and operation to optimize its operation for this purpose. The success of this apparatus has been demonstrated in recent publications on positron scattering from helium. ${ }^{8,9}$

The beamline is based on ideas developed in San Diego by Cliff Surko. A moderated ${ }^{22} \mathrm{Na}$ source provides an initial beam of low energy positrons, which are magnetically confined in the radial direction and guided into a Surko trap. ${ }^{2}$ Positrons in the trap are cooled to room temperature before being released as a high resolution pulsed beam. ${ }^{3}$ After interacting with the target gas of choice, the energy distribution of the beam is analyzed and scattering cross sections are calculated. ${ }^{10}$

The beamline is divided into several sections: the source/ moderator stage, the trap stage, the scattering apparatus, and the detection system. Each section and its operation will be explained in detail in Secs. II-VI.

\section{SOURCE AND MODERATOR}

The moderator stage of the apparatus was purchased from First Point Scientific, Inc., and uses solid neon as the moderator material. ${ }^{11}$ The ${ }^{22} \mathrm{Na}$ source is mounted on top of a low temperature cold head $(>6 \mathrm{~K})$ and primary shielding from high energy $(1.27 \mathrm{MeV})$ prompt gamma rays is provided by Elkonite blocks that surround the source and form part of the coldhead assembly. Pumping is provided by a 60 1/s turbomolecular pump and by a $100 \mathrm{l} / \mathrm{s}$ ion pump. The vacuum chamber containing the cold head and radioactive source is housed, in turn, in a cylindrical storage container filled with lead shots to provide further protection from gamma radiation. Under clean, high vacuum conditions, the low energy positron current, which is an indicator of the state of the moderator, decays at a rate of about 10\%/day. However, when exposed to the trap gases from the next stage of the apparatus (as is the case in normal operation), the moderated beam typically loses about half its strength in $24 \mathrm{~h}$.

The magnetic field at the positron source is approximately $80 \mathrm{G}$ and solenoid coils located along the vacuum chamber guide the beam to the next stage of the apparatus. A bias potential of up to $100 \mathrm{~V}$ can be applied to the moderator, defining the initial energy of the positron beam.

The combination of a neon moderator and a $50 \mathrm{mCi}$ source produces a flux of up to $10 \times 10^{6}$ positrons/s (the current source strength is approximately $25 \mathrm{mCi}$ ). From time to time the coldhead is unable to maintain the sub-10-K temperatures needed to form solid neon moderators and argon moderators have been used instead. ${ }^{11}$ The moderated beam intensity is approximately halved when using an argon moderator.

\section{POSITRON TRAPPING AND COLD PULSE FORMATION}

The positron beam emanating from the moderator is directed into the trap which is contained in a uniform (to better than $5 \%$ ) magnetic field of $\sim 530 \mathrm{G}$. The trap consists of nine electrodes of varying lengths and diameters and it is shown schematically in Fig. 1. The first three electrodes have internal diameters of $1 \mathrm{~cm}$ and lengths of 2,6 , and $6 \mathrm{~cm}$, respectively. These electrodes are separated by insulating spacers that provide electrical isolation and form part of a mechanically sealed cylindrical unit. Electrodes 4-8 are 


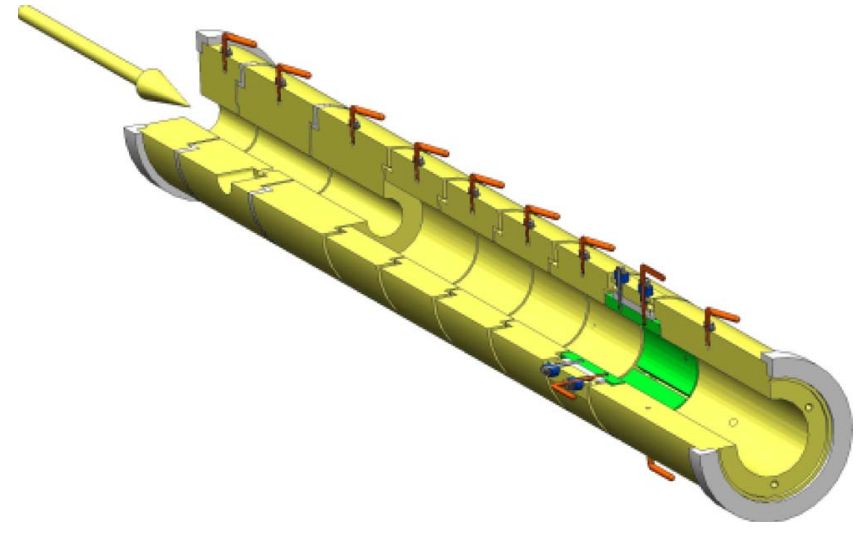

FIG. 1. (Color online) Schematic of the electrode arrangement used for the Surko trap. The arrow shows the direction of the positrons into the trap.

geometrically identical, having an internal diameter of $2 \mathrm{~cm}$ and a length of $3 \mathrm{~cm}$. One of these is segmented to provide future capability for the inclusion of a "rotating wall" to radially compress the trapped positron cloud. ${ }^{12}$ The position of the segmented electrode in the trap can be changed depending on the desired final configuration. The final electrode in the assembly, electrode 9 , has an internal diameter of $2 \mathrm{~cm}$ and a length of $6 \mathrm{~cm}$. These larger diameter electrodes are also electrically isolated from each other, using ruby ball spacers, but do not form a sealed unit. The trap assembly is housed inside a cylindrical canister.

Nitrogen gas is admitted directly into the smaller diameter section of the trap, electrodes 1-3, and diffuses into the larger diameter section, electrodes 4-9. The pressure of the nitrogen at the admission point is approximately 1 mTorr, while the pressure in the larger diameter region is about an order of magnitude lower. $\mathrm{CF}_{4}$ gas is admitted through the canister at the opposite end of the trap, diffusing into the larger diameter section with a pressure of approximately 0.01 mTorr. These two gases provide energy loss mechanisms by which the positrons are trapped and cooled, as a result of collisions, in the final section of the trap. ${ }^{2}$ Gas pressures in the final stage of the trap are much higher than for a conventional three-stage Surko trap, which results in a much shorter lifetime for the trapped positrons. As the trap typically operates in a fast cycling mode, this does not present a disadvantage for the intended operation of this apparatus. More detail on this is presented in Sec. IV.

Pumping of the trap section is performed using two 520 $1 / \mathrm{s}$ turbopumps, located in end stations at either end of the trap. Differential pumping tubes between the trap and the pumps reduce the pressure in the end stations when the trap is operational. In particular, at the upstream end of the trap, this reduces the contamination of the neon moderator from the nitrogen buffer gas. The base pressure in the trap section is $<3 \times 10^{-10}$ Torr and, when the trap gases are admitted and the trap is in operation, the pressures are typically 8.5 $\times 10^{-7}$ Torr at the entry to the trap and $7.9 \times 10^{-6}$ Torr at the exit to the trap. While the pumping speeds are much smaller than other Surko trap systems, which typically use cryopumps, the advantage of using turbopumps in the present apparatus is that it will be able to be used for experiments on helium, with the intention of establishing bench-
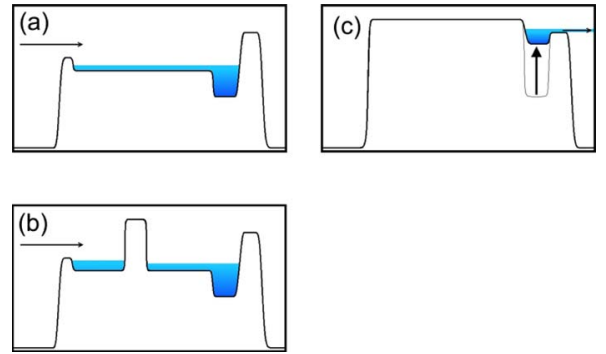

FIG. 2. (Color online) Potential arrangement on the electrodes for the various stages of trap operation: (a) trapping phase, (b) cooling phase, and (c) dump phase. The horizontal arrows show the direction of the positron beam into the trap and the solid line represents the potentials applied using the electrode structure from Fig. 1. The shaded area represents the trapped positrons.

mark cross section sets for positron scattering for a target which is relatively tractable for theoretical calculations with a high degree of accuracy. ${ }^{13}$

The magnetic field at the trap stage of the experiment is provided by a solenoid which has been constructed in-house. Electric potentials are applied to the trap electrodes using a combination of 16-bit digital-to-analog converters and fast, high voltage amplifiers. This allows the entire experiment to be operated under computer control. The entire system has very low electrical noise, $<3 \mathrm{mV} \mathrm{rms}$, and is capable of high slew rates, up to $30 \mathrm{~V} / \mu \mathrm{s}$, allowing for rapid trap cycling without compromising the energy resolution of the final positron pulse.

\section{TRAP OPERATION}

The trap is operated in a fast cycling mode to both minimize the effect of the low, trapped positron lifetime and to keep the number of positrons in each pulse relatively low so as to avoid saturation of the detection system. A typical trap cycle consists of three stages: a load stage, a cooling stage, where positrons are allowed to cool via collisions with the buffer gas, and a dumping stage where the cooled positrons are released from the trap before starting a new cycle. These configurations are illustrated in Figs. 2(a)-2(c), respectively. During the cooling and dumping stages [Figs. 2(b) and 2(c)], the trap is configured so that positrons can still be trapped in the first stage, enhancing the effective trapping efficiency and overall positron throughput in the system.

The throughput is governed by two main parameters: the number of positrons from the moderated beam that can be transferred into the trap region with the desired energy ("trappable" positrons) and the trapping efficiency. The number of trappable positrons is determined by the ratio of the magnetic field at the moderator to the field at electrode 1 of the trap. This is due to the partitioning of energy into the perpendicular and parallel components for charged particles in a magnetic field, $E_{\perp}$ and $E_{\|}$, respectively. Cylindrical electrodes with their axes parallel to that of the magnetic field will only couple to the $E_{\|}$component of the positron energy. As the magnetic moment $\left(E_{\perp} / B\right)$ is conserved, an increase in magnetic field will also increase the energy spread in the parallel component of the beam (this effect has been used in experiments to measure inelastic scattering cross sections)., 
The ratio of the magnetic fields between the moderator and trap in the current experiment is approximately 0.15 , leading to an increase in energy spread by a factor of approximately 6.5 in the $E_{\|}$component of the beam. The inherent energy of the positron beam spread using a neon moderator is around $1.5 \mathrm{eV}$, meaning that the energy spread in this component at the trap is $\sim 10 \mathrm{eV}$. In practical terms, this limits trappable moderated positrons in the trap to $40 \%-60 \%$ of the initial moderated positron beam. With the present design for the operation and cooling of the solenoids providing the magnetic fields, improvements in the transfer are not achievable until new solenoids have been designed and put into place.

The trapping efficiency can be defined as the probability of trapping a positron from the moderated beam during one trap cycle. Most trapping occurs during the loading stage with $\sim 90 \%$ of the trapped positrons collected over $75 \%$ of the cycle. The potentials of the trap are adjusted to maximize the efficiency by tuning to the excitation of the $a^{1} \Pi$ state of $\mathrm{N}_{2} \cdot{ }^{14,15}$ A maximum trapping efficiency of $10 \%$ has been obtained with the present apparatus, about half the reported peak efficiency of other systems. ${ }^{6,7}$ Further investigation of this difference will hopefully lead to an improved performance of the trap in the future.

Cooling of the positrons to the gas temperature takes place in electrode 8 by excitation of vibrational and rotational modes of the $\mathrm{N}_{2}$ and $\mathrm{CF}_{4}$ buffer gases. ${ }^{2,16}$ The throughput of positrons in the system is dependent on the ratio of the cooling time to the loading time. Longer cooling times result in lower positron signal, as positrons are lost either through annihilation or cross field transport from the trap, although improved energy resolution is achieved with longer cooling times. Thus different experimental requirements often require the balance of these two competing factors.

While the pressure profile in the trap is a two stage, the trap operates as a quasi-three-stage trap, as shown in Fig. 2. It was found that the positron lifetime in the trap was significantly shorter using a two-stage configuration, severely limiting the ability to cool the positrons to a sufficiently low temperature. This lifetime was substantially improved by changing to a three-stage configuration. Lifetimes can be measured by trapping for a fixed time and then holding the positrons in the third stage for varying times before dumping the pulse and measuring the resultant signal. The curve produced in this manner, shown in Fig. 3, can then be fitted to a simple exponential decay. From this procedure, a lifetime of $34 \mathrm{~ms}$ was obtained for the third stage of the trap, under normal operating conditions. This lifetime is found to scale linearly with the nitrogen pressure in the trap. While the lifetime would ideally be limited only by annihilation on the background buffer gas, it appears to be somewhat lower than expected (the corresponding lifetime from the operation of the San Diego experiment would be about $300 \mathrm{~ms}$, scaled for the differing pressures in the final stage). ${ }^{3}$ This is a possible indication that anomalous radial transport of the positrons also plays a role. This is likely the limiting factor in the trapped positron lifetime in the present apparatus and should be able to be improved with the introduction of the rotating wall. ${ }^{7}$ Another possibility is that the pressure in the third

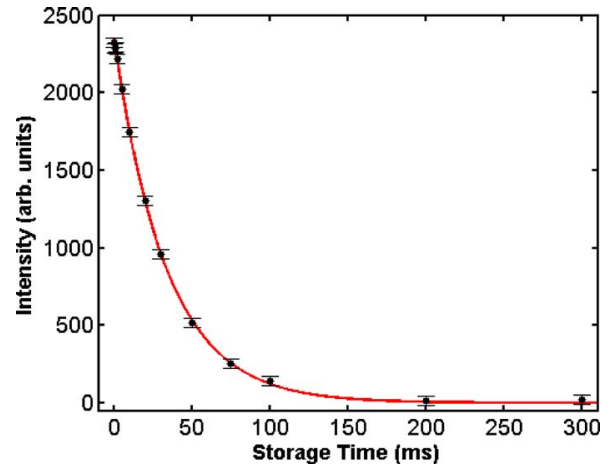

FIG. 3. (Color online) Positron signal as a function of time confined in the third stage. An exponential fit to the data (shown as the solid line) gives a lifetime of $34 \mathrm{~ms}$.

stage of the present trap has been underestimated, although this is unlikely to account for the entire difference. An attempt was also made to analyze the lifetime in the trap using the method suggested by Clarke et al.; ${ }^{6}$ however, this was unable to be applied in a satisfactory manner, suggesting that the loss rate in the present apparatus cannot be modeled in the same way. This may be accounted for by differences in the operation of the trap or possibly by different mechanisms dominating the loss in each case.

\section{SCATTERING APPARATUS}

After the trap there are two further vacuum chambers, containing a gas cell, and the retarding potential analyzer (RPA) and the multichannel plate detector (MCP). A 520 1/s turbopump achieves differential pumping between the trap and the gas cell chambers. Another turbopump (210 l/s) lies between the gas cell and the final vacuum chamber. The gas cell is $200 \mathrm{~mm}$ long with an internal diameter of $70 \mathrm{~mm}$ and entrance and exit apertures of $5 \mathrm{~mm}$. It is located in a solenoid producing a magnetic field equal in magnitude to that of the trap. Small mesh cylinders, placed at each end of the cell, keep the potential immediately outside the cell the same as that inside. This ensures that scattering from the small region of increased gas pressure, relative to the vacuum, at the exit of the cell is at the same energy as inside the cell, thus minimizing end effects.

Immediately before the cell assembly, there is another mesh cylinder, $15 \mathrm{~mm}$ in diameter and $45 \mathrm{~mm}$ long, which can be set to a potential independent of that of the gas cell. This electrode acts as a RPA and serves to discriminate against any scattering that may occur in the region between the trap exit and the gas cell due to background gases escaping from the trap. Positrons that have lost any parallel energy in transmission from the trap to the cell (from elastic or inelastic collisions with background gas) are rejected at this stage, preventing background scattering from influencing the cross section measurement. The cell is surrounded by a Teflon collar, which prevents trap gases diffusing downstream from the gas cell and eliminates scattering from the trap gases after the cell as a potential problem in the measurements. The gas cell assembly is shown in Fig. 4.

The $5 \mathrm{~mm}$ apertures of the cell are too small to allow the entire positron pulse to pass through and there is some loss 


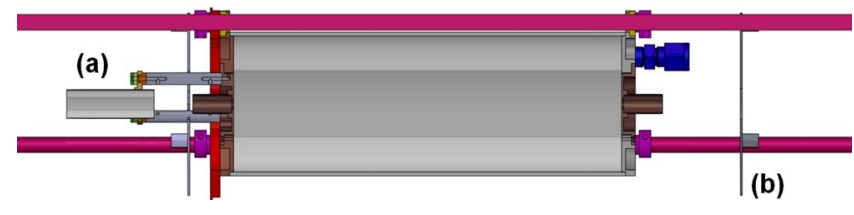

FIG. 4. (Color online) Schematic of the gas cell used for experiments. The first RPA is also shown (a), along with a shielding mesh that prevents voltage penetration from the gas cell to the positron beam transport region (b).

of beam intensity. This is minimized by ensuring that the highest flux portion of the pulsed beam (i.e., the center, see Fig. 5) is in line with the apertures, resulting in $40 \%-50 \%$ transmission.

Gas is admitted to the cell through a manually controlled needle valve and the pressure is monitored using a baratron gauge. The gauge used is a high accuracy pressure transducer with a full range of 1 Torr and a measurement accuracy of $\pm 0.05 \%$. The gauge is regulated to operate at $45{ }^{\circ} \mathrm{C}$, while the gas cell has a temperature of $\sim 30{ }^{\circ} \mathrm{C}$ so pressure readings must be scaled to account for thermal transpiration effects, and this correction is approximately $2 \%$ of the measured value. Typical operating pressures for helium gas experiments are between 5 and 25 mTorr with lower pressures anticipated for most other targets that will be studied. The use of turbopumps rather than cryopumps, more commonly used in Surko trap systems, ${ }^{2,6,7}$ allows the study of helium as a scattering target. Helium is an important target as its study provides a better understanding of the positron scattering problem with high accuracy, as is the case in electron scattering. Positron-helium scattering will form an important test bed for the development of both theory and experimental techniques. ${ }^{13}$

A final, separately adjustable, solenoid surrounds the last chamber containing the RPA which is $70 \mathrm{~mm}$ in diameter and $230 \mathrm{~mm}$ long. The RPA is used to analyze the energy $\left(E_{\|}\right)$ spread in the beam after passage through the gas cell and thus to enable the measurement of the scattering cross sections of interest. ${ }^{5}$

The energy of the positron interaction is defined by the potential difference between the final electrode of the trap, which determines the transport energy of the pulse and the potential of the gas cell. In practice, a measurement of the potential at which the gas cell cuts off the positron beam provides the zero energy of the gas cell, and the width of the cut off curve is determined by the energy resolution of the beam. ${ }^{3}$ Using 16 bit, $\pm 10 \mathrm{~V}$ digital-to-analog converters, combined with a ten times amplifier, voltage steps can be as small as $3 \mathrm{mV}$, providing sufficient ability to perform high resolution measurements of scattering processes.

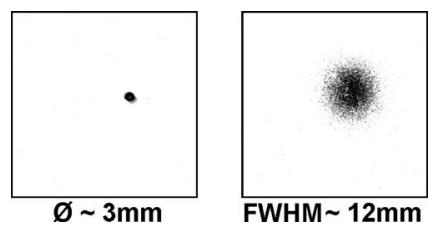

FIG. 5. Spatial distribution of the moderated (left) and trapped (right) positron beams in a $500 \mathrm{G}$ field.

\section{POSITRON DETECTION}

Detection of the positrons is achieved using a double stack MCP assembly combined with a capacitively coupled transimpedance amplifier. This detection arrangement amplifies the positron current incident upon the MCP and the amplifier converts the current pulse to a voltage pulse which is then stored digitally. Subsequently, this pulse is integrated to measure the signal intensity, which is proportional to the number of positrons in each pulse.

Signals are averaged over multiple positron pulses to achieve sufficient statistical accuracy. Also incorporated into the MCP assembly is a phosphor screen anode, which allows imaging of the positron beam for alignment and optimization purposes. This detection scheme differs from the conventional use of a NaI crystal to detect the annihilation gamma rays when the positrons hit the end of the apparatus. It has the advantage that detection of almost all the positrons in the pulse is possible, avoiding being limited by solid angle considerations in the case of the detection of annihilation gamma rays. This will lead to much higher data taking rates for a standard system of equivalent source strength and trap efficiency.

\section{PULSE ANALYSIS}

Analysis of the pulsed positron beam is undertaken in several ways. First, the beam can be imaged using the phosphor screen to give information about size and spatial distribution of the positron cloud. The pulsed beam can also be directed to a beam flag (a $72 \mathrm{~mm}$ diameter, gold-plated copper disk) located approximately $40 \mathrm{~cm}$ after the trap. This allows a measurement of the effective current of positrons which can be compared to the incident moderated beam. By knowing the frequency of the trap cycle, the number of positrons per pulse can be calculated. The timing distribution of the positron pulse can also be measured using the signal from the MCP and transimpedance amplifier. To a certain extent this can be thought of as an indicator for the energy resolution of the beam, as a higher energy spread of positrons in the trap leads to a greater spread in arrival times in the pulse. Finally, the energy spread of the beam can be analyzed using a RPA located downstream from the trap in a separate magnetic field coil. The principles behind this type of measurement are explained in detail elsewhere. ${ }^{5}$ Figure 5 shows the spatial distribution of the initial moderated positron beam and the final pulse from the trap. The moderated beam is annular in shape (a result of the source stage geometry) with an outer diameter of about $3 \mathrm{~mm}$ in a $530 \mathrm{G}$ field. The diameter of the trapped beam is $\sim 15 \mathrm{~mm}$ in a field of the same strength.

Throughput in the system can be as high as $5 \times 10^{5}$ cooled positrons/s with a $50 \mathrm{mCi}$ source. Individual pulses typically consist of $100-500$ positrons, with a pulse frequency ranging from 100 to $300 \mathrm{~Hz}$. The best beam energy width achieved to date has been $54 \mathrm{meV}$, shown in Fig. 6, somewhat higher than the $18 \mathrm{meV}$ beams reported elsewhere. ${ }^{3}$ Typical operation has an energy spread of 60 $\mathrm{meV}$, although early measurements ${ }^{8}$ had an energy spread somewhat higher than this. Increasing the cooling time or the 


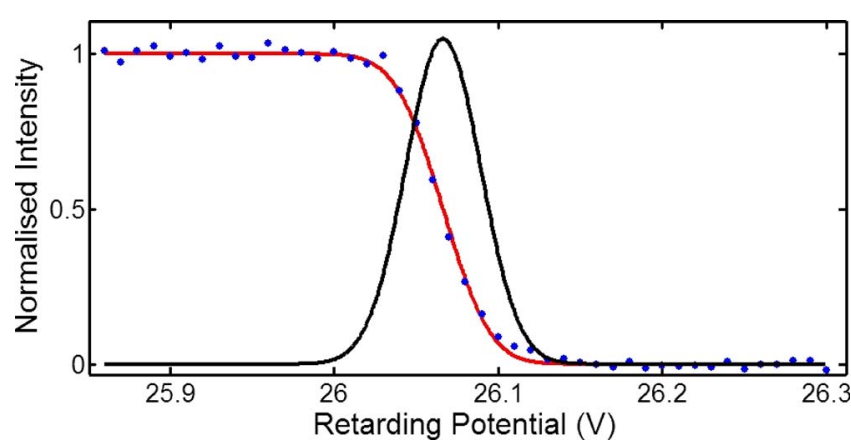

FIG. 6. (Color online) Cutoff curve for the trapped positron beam. Points are the experimental data and the curve through the points corresponds to a Gaussian energy distribution, also show on the plot. The energy of the beam in this case is $26.07 \mathrm{eV}$ and the energy resolution is $54 \mathrm{meV}$ (full width at half maximum).

pressure of the cooling gases (mostly $\mathrm{CF}_{4}$ ) in the final stage does not improve this, and electrical noise on the electrodes has also been investigated as a possible limitation for the resolution. However, the reasons for the higher than expected energy spread remain unclear although it is anticipated that the resolution can be improved upon in the future.

\section{CONCLUDING REMARKS}

Using the techniques outlined above we have succeeded in measuring absolute positron- $\mathrm{He}$ total cross sections for 1-15 eV using a beam with a resolution of $80 \mathrm{meV}^{8}$, and preliminary differential elastic scattering cross sections for 5 , 10 , and $15 \mathrm{eV},{ }^{9}$ demonstrating the viability of this experiment. Future work will concentrate on low energy scattering processes, making use of the high resolution of this positron beam combined with techniques developed for scattering in a strong magnetic field. ${ }^{5}$ Measurements of both threshold effects and state selective excitation processes will be possible, as well as benchmark measurements for simple scattering systems, ${ }^{13,17}$ leading to a new and deeper understanding of the physics of positron collisions.

\section{ACKNOWLEDGMENTS}

The authors would like to acknowledge the technical assistance of Graeme Cornish, who has provided an invaluable amount of work in the design and construction of the experiment outlined in this paper. Thanks also to Steve Battisson for his contributions. Professor Cliff Surko and Professor Mike Charlton have provided much helpful advice during the development of this apparatus. Finally, the authors would like to acknowledge the Australian Research Council for funding support, through the Centre of Excellence program, as well as the LIEF and Discovery funding programs.

\footnotetext{
${ }^{1}$ See, for example, M. Charlton and J. W. Humberston, Positron Physics (Cambridge University Press, Cambridge, 2001) and references therein.

${ }^{2}$ T. Murphy and C. M. Surko, Phys. Rev. A 46, 5696 (1992).

${ }^{3}$ S. J. Gilbert, C. Kurz, R. G. Greaves, and C. M. Surko, Appl. Phys. Lett. 70, 1944 (1997)

${ }^{4}$ J. P. Sullivan, S. J. Gilbert, and C. M. Surko, Phys. Rev. Lett. 86, 1494 (2001).

${ }^{5}$ J. P. Sullivan, J. P. Marler, S. J. Gilbert, S. J. Buckman, and C. M. Surko, Phys. Rev. Lett. 87, 073201 (2001).

${ }^{6}$ J. Clarke, D. P. van der Werf, B. Griffiths, D. C. S. Beddows, M. Charlton, H. H. Telle, and P. R. Watkeys, Rev. Sci. Instrum. 77, 063302 (2006).

${ }^{7}$ D. B. Cassidy, S. H. M. Deng, R. G. Greaves, and A. P. Mills, Jr., Rev. Sci. Instrum. 77, 073106 (2006).

${ }^{8}$ J. P. Sullivan, C. Makochekanwa, A. Jones, P. Caradonna, and S. J. Buckman, J. Phys. B 41, 081001 (2008).

${ }^{9}$ J. P. Sullivan, A. Jones, P. Caradonna, C. Makochekanwa, and S. J. Buckman, Nucl. Instrum. Methods Phys. Res. B 266, 384 (2008).

${ }^{10}$ J. P. Sullivan, S. J. Gilbert, J. P. Marler, R. G. Greaves, S. J. Buckman, and C. M. Surko, Phys. Rev. A 66, 042708 (2002).

${ }^{11}$ A. P. Mills, Jr. and E. M. Gullickson, Appl. Phys. Lett. 49, 1121 (1986).

${ }^{12}$ R. G. Greaves and J. M. Moxom, Phys. Plasmas 15, 072304 (2008).

${ }^{13}$ S. J. Buckman and J. P. Sullivan, Nucl. Instrum. Methods Phys. Res. B 247, 5 (2006).

${ }^{14}$ C. M. Surko, M. Laventhal, and A. Passner, Phys. Rev. Lett. 62, 901 (1989).

${ }^{15}$ J. P. Marler and C. M. Surko, Phys. Rev. A 72, 062713 (2005).

${ }^{16}$ R. G. Greaves and C. M. Surko, Phys. Rev. Lett. 85, 1883 (2000).

${ }^{17}$ C. M. Surko, G. F. Gribakin, and S. J. Buckman, J. Phys. B 38, R57 (2005)
} 